\title{
THE SPECIFIC INDUCTIVE CAPACITY OF CERTAIN METALS.
}

By FERNANDO SANFORD.

$A \mathrm{~N}$ electron moving in an elliptical or circular orbit around a central A positive charge must have an acceleration toward the center of its orbit proportional to the ratio of its mean orbital radius to the square of its periodic time, hence its centripetal force may be written $F^{\prime} \propto\left(R / T^{2}\right)$, where $R$ is its orbital radius and $T$ its periodic time.

If such electron be a source of light, its wave-length will be proportional to its periodic time, hence we may write $F^{\prime} \propto\left(R / \lambda^{2}\right)$.

While we cannot know with certainty the orbital radii of the electrons revolving around different atoms, we may, at least, make plausible assumptions about them, and on the basis of these assumptions we may calculate the relative magnitude of the forces acting upon them.

In the spectral series which have been recognized in a considerable number of elements the wave-lengths converge toward some shortest possible wave-length according to a definite law. In the group of alkali metals three such spectral series are known for each element. In the one known as the principal series in each metal the wave-lengths toward which the series converges is less than the wave-length of any known line in the spectrum of the respective metal. It accordingly seems a plausible assumption that no electron in any of these metals may revolve around the central charge at a distance less than the orbital radius corresponding to the convergence wave-length.

If we may assume the orbital radius corresponding to this convergence wave-length as the true radius of the atom, we may calculate the relative magnitudes of the positive charges of the atoms of this group when we know their atomic radii.

If instead of the wave-length of a given spectral line we use its vibration frequency, we may write $F^{\prime} \propto R n^{2}$, where $n$ is the vibration frequency. Since $F^{\prime}$ represents the centripetal force upon an electron at distance $R$ from the central charge, if we wish to find what this centripetal force would be at unit distance from the central charge we must multiply $F^{\prime}$ by $R^{2}$, and we have $F \propto R^{3} n^{2}$. Since $R^{3} \propto V$, the atomic volume, we may write $F \propto V n^{2}$.

In Ann. d. Phys., 4I, 524, Heydweiller has given a number of atomic diameters calculated from the volume of a gram-ion of the element in an 
infinitely dilute water solution. If we assume that these diameters are equal to the orbital diameters of the innermost possible electron in the spectral series of the respective atoms, we may calculate the attraction of each central atom for an electron at unit distance. If the central charges of the atoms are equal, we should expect these forces also to be equal.

In the table below are given Heydweiller's atomic diameters, the convergence numbers and the force $F=V n^{2}$ for all the elements whose atomic diameters are known and for which spectral series are given in Kayser's Handbuch. It should be remembered that it is only in the alkali metals that the convergence numbers used are regarded as belonging to the principal series of the elements. It is accordingly only to these elements that we can apply the above argument with certainty.

\begin{tabular}{|c|c|c|c|}
\hline Element. & Convergence Number. & Atomic Diameter. & $F$ \\
\hline Li $\ldots \ldots \ldots$ & 4,358 & 1.75 & 1,016 \\
\hline $\mathrm{Na} \ldots$ & 4,154 & 1.93 & 1,240 \\
\hline K.... & 3,509 & 2.38 & 1,662 \\
\hline Rb... & 3,376 & 2.62 & 2,059 \\
\hline Cs.... & 3,151 & 2.93 & 2,500 \\
\hline $\mathrm{Mg}$. & 3,984 & 2.01 & 1,285 \\
\hline Ca.. & 4,041 & 2.43 & 1,652 \\
\hline Sr... & 3,106 & 2.62 & 1,737 \\
\hline $\mathrm{Ag} .$. & 3,071 & 2.74 & 1,952 \\
\hline $\mathrm{Cu} \ldots .$. & 3,159 & 2.38 & 1,348 \\
\hline $\mathrm{Zn}$. & 4,295 & 2.38 & 2,500 \\
\hline Cd. . & 4,080 & 2.70 & 3,280 \\
\hline T1....... & 4,154 & 3.27 & 6,050 \\
\hline
\end{tabular}

It is seen that the above values of $F$ are not equal, and that accordingly the elements apparently do not have equal central charges. There is a suggestion of regularity in the increase of $F$ from one atom to the next in the same group, so that if these values of $F$ be plotted against the serial atomic numbers of Rydberg a marked regularity is seen in the group of the alkali metals, but not in the whole list.

It has previously been shown by the present writer that the atomic charges calculated from the mobility of ions in electrolysis vary with the serial numbers at a constant rate, but their relative values are not the same as the relative values of the central forces here calculated. This would seem to indicate, if our conclusions are correct, that the atoms must attract their electrons with forces which are not proportional to their central charges, and accordingly that they must have different specific inductive capacities. 
Voc. VIII.] SPECIFIC INDUCTION CAPACITY OF CERTAIN METALS.
No.x.

Since the attraction between an electron and a positive charge at unit distance is given by the equation $F=Q e / k$, where $Q$ is the magnitude of the positive charge and $k$ is the specific inductive capacity, and since the charge $e$ is the same for all electrons, we may write $k \propto Q / F$. Calculated in this way from the charges of the ions in electrolysis, the relative specific inductive capacities of the above elements are given in the following table.

\begin{tabular}{|c|c|c|c|}
\hline Element. & $F$ & $Q$ & $k$ \\
\hline Li $\ldots \ldots \ldots \ldots$ & 1,016 & 3.65 & 359 \\
\hline$\ldots \ldots \ldots$ & 1,240 & 15.7 & 1,270 \\
\hline K. . & 1,662 & 39.6 & 2,380 \\
\hline $\mathrm{Rb} \ldots \ldots \ldots \ldots$ & 2,059 & 91.2 & 4,370 \\
\hline Cs.......... & 2,500 & 142 & 5,670 \\
\hline $\mathrm{Mg}$. & 1,285 & 17.4 & 1,340 \\
\hline $\mathrm{Ca} \ldots \ldots \ldots \ldots$ & 1,652 & 32.5 & 1,960 \\
\hline Sr.......... & 1,737 & 71.5 & 4,120 \\
\hline $\mathrm{Ag}$. & 1,952 & 91.6 & 4,660 \\
\hline $\mathrm{Cu} \ldots \ldots \ldots \ldots$ & 1,348 & 46.7 & 3,460 \\
\hline $\mathrm{Zn}$. & 2,500 & 47.6 & 1,900 \\
\hline $\mathrm{Cd} \ldots$ & 3,280 & 83.8 & 2,550 \\
\hline T1.......... & 6,050 & 199.4 & 3,500 \\
\hline
\end{tabular}

That the above numbers, which are here assumed to represent the relative specific inductive capacities of the elements, are definitely related to other atomic constants may be shown by plotting them against Rydberg's serial numbers. This is done in the accompanying curve (Fig. I), and it will be seen that for ten of the thirteen elements given in the above table the calculated specific inductive capacities bear a constant relation to the serial numbers, while for the other three they are just half the values suggested by the curve.

This could not have been inferred from our knowledge of the spectral series, since there is no reason to conclude that for all the elements here given except the alkal metals there could not be other series with smaller convergence radii than those here used. Thus in the alkali metals the subordinate series have convergence radii I.37 times as great as those here used.

The question as to whether the numbers here calculated actually represent the relative specific inductive capacities of their respective atoms can only be answered inferentially from the variation of properties which in non-metallic elements vary with specific inductive capacity. A comparison with the melting points of the alkali metals and with the 
refraction constants of all the metals here used show quite as close an agreement as these properties show with the measured specific inductive capacity of other elements. The relation of the specific inductive capa-

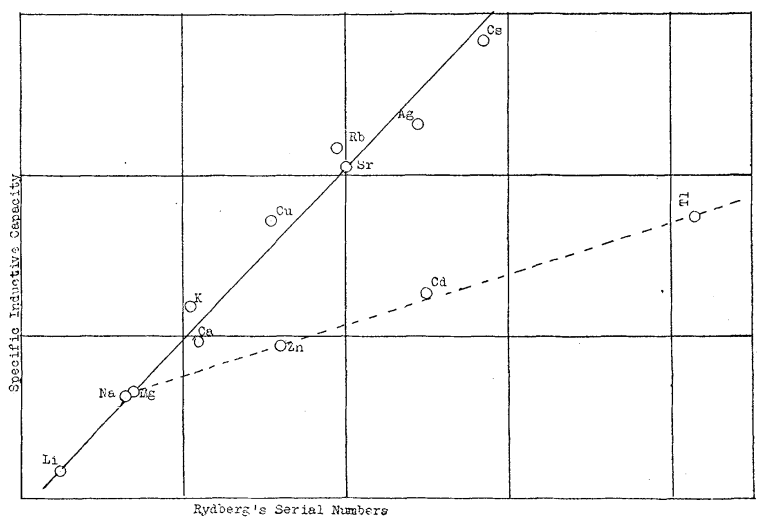

Fig. 1.

cities calculated above to Heydweiller's refraction constants for the same elements is shown in Fig. 2.

A further justification for the above values of specific inductive capacity may, perhaps, be found in their relations to Planck's law. Thus it has been found that the energy of electrons set free by the action of ultra-violet light

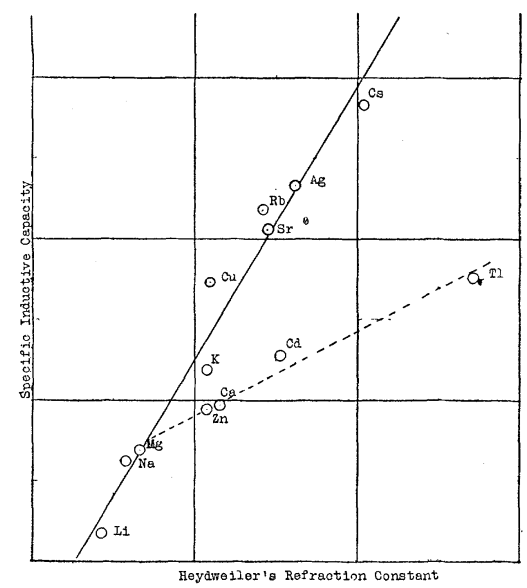

Fig. 2.

on different metals is independent of the nature of the metal and proportional to the frequency of the incident light. It seems very probable that the energy of the expelled electrons is the same as their orbital energy at the 
instant of expulsion, and that their orbital frequency was before expulsion the same as the frequency of the incident light. If this is the case, the orbital energy of an electron at the instant of its expulsion would seem to have been $E=n h$, where $n$ was its orbital frequency and $h$ is Planck's constant.

The orbital energy of an electron is also given by the equation $E=m v^{2} / 2$, hence $m v^{2} / 2=n h$ and $v^{2} \propto n \propto \mathrm{I} / T$, where $T$ is the periodic time. But $T \propto R / v$, where $R$ is the orbital radius, hence $v^{2} \propto v / R$, and $v \propto \mathrm{I} / R$. Accordingly, in order that the energy of revolving electrons may increase as their frequency, their orbital velocity must vary inversely as the orbital radius.

The centrifugal force of such an electron is $F^{\prime}=v^{2} / R$. Substituting for $v^{2}$ its equivalent $\mathrm{I} / R^{2}$, we have $F^{\prime} \propto \mathrm{I} / R^{3}$. Hence, in order that the orbital energy of different electrons may vary as their frequencies, the forces between them and their central positive charges must vary as the inverse third power of their orbital radii.

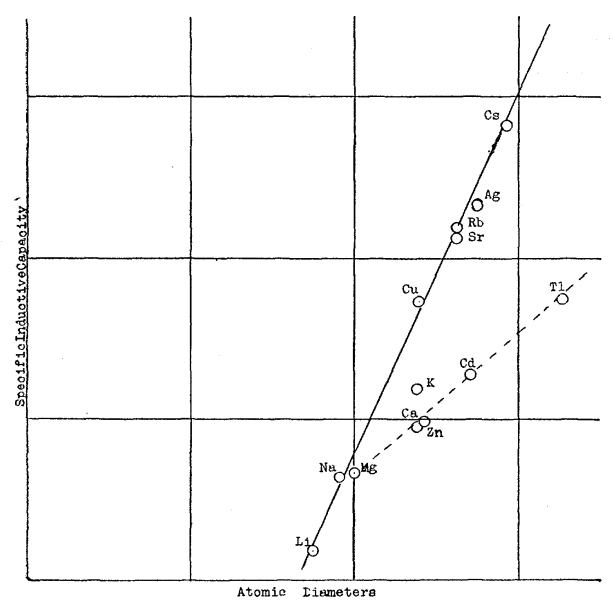

Fig. 3.

The electric attraction between an electron and its central charge must vary as the inverse second power of its orbital radius and also inversely as the specific inductive capacity of the intervening medium, $i$. e.; $F^{\prime} \propto \mathrm{I} / k R^{2}$, hence for $k \propto R, F^{\prime} \propto \mathrm{I} / R^{3}$.

That the specific inductive capacities of the different atoms here considered do vary approximately as the respective atomic radii with the same exceptions as in the case of the serial numbers is shown in the table below and in Fig. 3. This would make Planck's equation apply to different frequencies when these are caused by changing from one atom to 
another of a different element. In the case of any one element the difference in the orbital radii for the different electrons which give the shorter wave-lengths is very small. Thus for the lines for which $N=9$ and $N=$ ro in Balmer's formula while the frequency would differ by about 2 per cent., the difference in orbital radii would be less than 0.5 per cent.

Heydweiller's atomic diameters and specific inductive capacity.

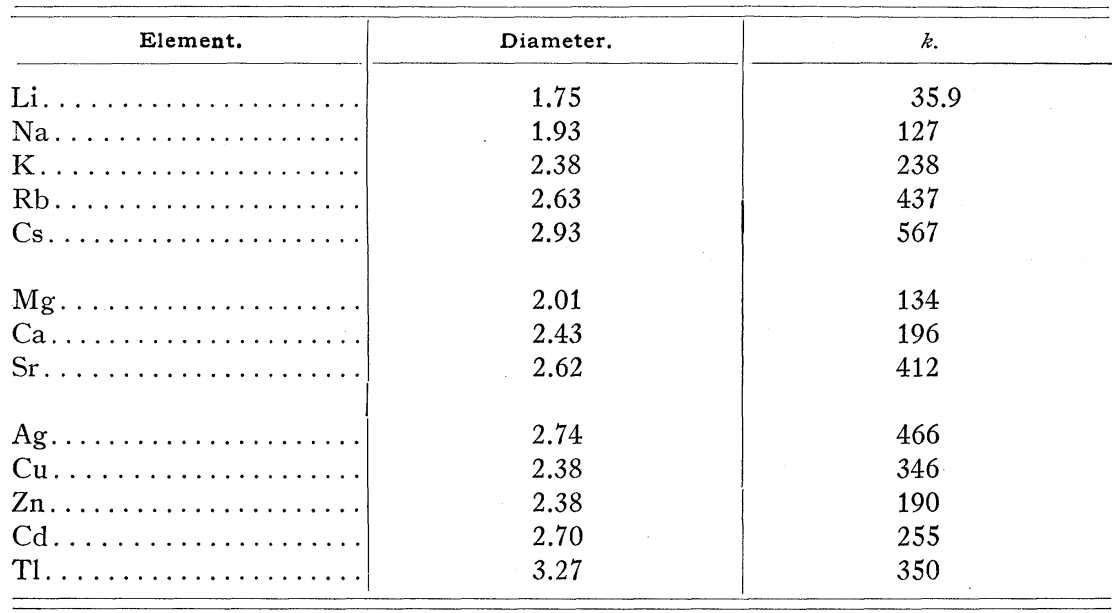

STANFORD UNIVERSITY,

March 4, 1916. 